\title{
25.-27.9.2014: Österreichischer Anwaltstag in Hall/Tirol
}

\author{
Dr. Geertje Tutschka \\ Rechtsanwältin, CLP: Coaching for Legal Professionals, \\ Salzburg
}

Vom 25. bis 27. September 2014 fand in Hall/Tirol der alljährliche Anwaltstag der österreichischen Rechtsanwaltschaft (ÖRAK) statt.

Nach dem Begrüßungsabend im Parkhotel folgte die Festliche Eröffnung im Großen Saal des Salzlagers Hall am Freitag nach Grußworten der Bürgermeisterin der Stadt Hall in Tirol Dr. Eva-Maria Posch und des Präsidenten der Tiroler Rechtsanwaltskammer Dr. Markus Heis durch den Präsidenten des Österreichischen Rechtsanwaltskammertages Dr. Rupert Wolff. Dr. Maria Berger, Richterin am Gerichtshof der Europäischen Union, schloss sich mit einer Festansprache zum Thema „Das Bild des Rechtsanwaltes in der Rechtsprechung des EuGH“ an. Die anschließende Podiumsdiskussion zum Thema „Geheimnisschutz oder schutzlos transparent " beinhaltete insbesondere das Impulsreferat von Dr. Maria Wittmann-Tiwald, der Vorsitzenden der Fachgruppe Grundrechte und interdisziplinärer Austausch der Vereinigung der österreichischen Richterinnen und Richter.

Während bei der feierlichen Eröffnung am Vormittag von den insgesamt sieben Festansprachen und Grußworten immerhin drei von weiblichen Repräsentantinnen gehalten wurden, stand bei der anschließenden Podiumsdiskussion am frühen Nachmittag lediglich eine Richterin den weiteren vier männlichen Impulsreferenten und dem Moderator Präsident Dr. Rupert Wolff gegenüber. Obwohl es hier bislang keine Referentinnenquote gibt, präsentierte sich der ÖRAK damit den Kolleginnen und Kollegen auf dem Österreichischen Anwaltstag erfreulicherweise mit einem relativ ausgewogenem Verhältnis von Referentinnen und Referenten.

Große Beachtung erfuhr die Präsentation der im ÖRAK seit Kurzem neugebildeten Arbeitsgruppe „Frau in der Rechtsanwaltschaft” mit der Vorsitzenden Rechtsanwältin Dr. Sonja Schröder mit dem Workshop „Jurist oder Juristin - (k)ein Unterschied" durch Dr. Geertje Tutschka von CLP, Coaching for legal professionals. Dr. Tutschka, die als deutsche Juristin sowohl bei der Rechtsanwaltskammer in Hamm in Nordrhein-Westfalen in Deutschland als auch bei der Salzburger Rechtsanwaltskammer als Europaanwältin zugelassen ist, arbeitet hauptsächlich als Coach und Trainer für Juristinnen und Juristen und Anwältinnen und Anwälte im deutschsprachigen Raum. Sie erarbei- tete mit den Teilnehmern die genderspezifischen Schlüsselqualifikationen des anwaltlichen Berufes sowie des entsprechenden Entwicklungspotenzials von Anwältinnen und präsentierte die aktuellen österreichischen Zahlen der Neuzulassungen zur Anwaltschaft von Frauen und Männern. Obwohl in den letzten Jahren erstmals doppelt so viele Anwältinnen zugelassen worden waren, stagniert ihr Anteil in der österreichischen Anwaltschaft seit Jahren unter 20 Prozent. Und das, obwohl in den JUS-Fakultäten der Universitäten als auch später bei der Konzipientenausbildung (fünfjährige Ausbildung zum Anwalt - in etwa vergleichbar mit dem Referendariat in Deutschland) ebenso viele weibliche wie männliche Studierende bzw. Konzipienten die Ausbildung durchlaufen und erfolgreich abschließen. Auch bilden die Zahlen in anderen juristischen Berufen - in der Justiz, in der Verwaltung oder in der Wirtschaft - lediglich das ausgewogene Verhältnis der Studierenden ab, nicht aber ein entsprechendes „Zuwandern” von Anwältinnen in diese Berufe.

\section{Erforscht der Anwalt sie auf „typisch männliche“ oder eher „typisch weibliche“ Eigenschaften?}

Dr. Tutschka erforschte die Ursachen für dieses Phänomen und ging in dem gut 60-minütigen Workshop „Jurist oder Juristin - (k)ein Unterschied“ der Frage nach, ob der anwaltliche Beruf möglicherweise „typisch männliche“ oder eher „typisch weibliche“ Eigenschaften erfordert oder ob es in Österreich im Gegensatz zu anderen europäischen Ländern spezifische Umstände gibt, die einer Neuzulassung von Anwältinnen entgegenstehen. Immerhin zeigen die Zahlen der Entwicklung des weiblichen Anteils an der Anwaltschaft in europäischen Vergleichsländern - wie beispielsweise in Deutschland, Frankreich und Schweden - ein eher ausgewogenes GenderVerhältnis.

Die sich anschließende Diskussion thematisierte lebhaft das in der Österreichischen Anwaltschaft bestehende Gender Gap und dessen Ursachen. Dabei konnten bereits einzelne spezifiziert werden sowie diverse wirksame Einzelmaßnahmen zur Förderung und Unterstützung der Anwältinnen auf Länderebene vorgestellt werden, wie beispielsweise ein Substitutionspool (eine Liste von kurzfristig einsetzbaren Vertretern), die Möglichkeit der vorübergehenden Befreiung von der Rentenversicherungspflicht oder die Einrichtung von Kinderbetreuungsmöglichkeiten. 
Im Übrigen war auch erstmals eine Kinderbetreuung während des Anwaltstages eingerichtet worden, die den Rechtsanwältinnen und Rechtsanwälten die Möglichkeit bot ihre Kinder pädagogisch betreuen zu lassen.

Obgleich „Gender Diversity im Anwaltsberuf” damit erstmals Thema eines Österreichischen Anwaltstags war und auch das Format eines Workshops beim Anwaltstag neu war, wurde das Angebot sehr gut angenommen. Bereits wenige Wochen nach der Veröffentlichung des Programms vor der Sommerpause war der Workshop mit 64 Teilnehmerinnen und Teilnehmern ausgebucht gewesen und zwar zu nahezu gleichen Teilen von Berufskolleginnen und -kollegen, unter ihnen der Präsident des ÖRAK Dr. Rupert Wolff, Vizepräsident Dr. Armenak Utudjian, Vizepräsident Dr. Josef Weixelbaum sowie zahlreiche Arbeitsgruppenmitglieder und mehrere Länderpräsidenten.

Die rege Diskussion zum Thema brachte Austausch und Sensibilisierung. Die schon bestehenden Maßnahmen und Einzelinitiativen zur Förderung und Unterstützung von Frauen im Anwaltsberuf sollen nun zusammengefasst und darüber einheitlich informiert werden, um noch größere Transparenz zu erzielen. Die erarbeiteten Vorschläge und Ideen werden mit einem Bericht im Österreichischen Anwaltsblatt im Dezember 2014 veröffentlicht werden. Die „Arbeitsgruppe Frau in der Anwaltschaft" des ÖRAK wird diese Vorschläge aufgreifen und zur Grundlage ihrer weiteren Arbeit machen. Wir dürfen also gespannt sein.

Der Anwaltstag der österreichischen Rechtsanwaltschaft wurde dann am Samstag mit der Präsidentenratssitzung, der ÖRAK-Vertreterversammlung und der Tagung der Präsidenten der Diszipliarräte erfolgreich beschlossen.

Vortragende Dr. Geertje Tutschka bietet all denjenigen, die nicht am Workshop teilnehmen konnten, die Möglichkeit, am Mittwoch, den 7. Januar 2015 um 18.00 Uhr den Workshop als Webinar im Internet nachzusehen.

Kostenfreie und unverbindliche Anmeldung für die Zusendung der Einwahldaten für das Webinar auf der Webseite unter www.coachingforlegals.com/service/webinare beim Webinar "Jurist oder Juristin“.

Die Einwahldaten werden Ihnen dann zugesandt.

\section{Männer \& Frauen in der österreichischen Anwaltschaft}

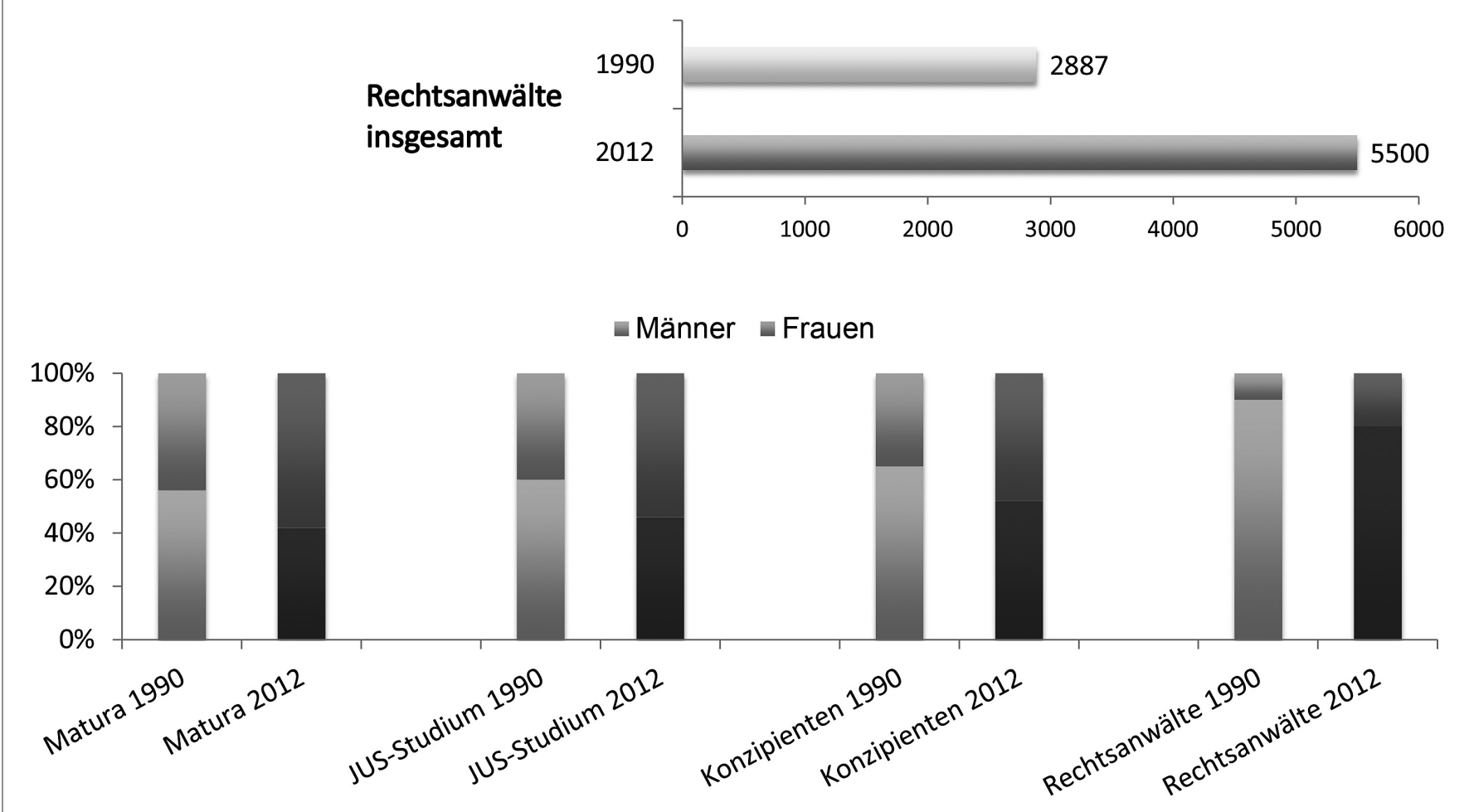

(C) Dr. Geertje Tutschka 